\title{
Synchrotron x-ray diffraction study of micro-patterns obtained by spatially selective hydrogenation of GaAsN
}

\author{
G. Ciatto, ${ }^{1, \text { a) }}$ G. Pettinari, ${ }^{2}$ N. Balakrishnan, ${ }^{3}$ F. Berenguer, ${ }^{1,4}$ A. Patanè,${ }^{3}$ S. Birindelli, ${ }^{5}$ \\ M. Felici, ${ }^{5}$ and A. Polimeni ${ }^{5}$ \\ ${ }^{1}$ Synchrotron SOLEIL, L'Orme des Merisiers, Saint-Aubin, BP48, 91192 Gif-sur-Yvette Cedex, France \\ ${ }^{2}$ CNR, Institute for Photonics and Nanotechnologies, via Cineto Romano 42, 00156 Roma, Italy \\ ${ }^{3}$ School of Physics and Astronomy, The University of Nottingham, NG7 2RD Nottingham, United Kingdom \\ ${ }^{4}$ IPANEMA CNRS, MCC, USR 3461, Synchrotron SOLEIL, BP48 Saint-Aubin, F-91192 Gif-sur-Yvette, France \\ ${ }^{5}$ Department of Physics, Sapienza Università di Roma, Piazzale A. Moro 2, 00185 Roma, Italy
}

(Received 11 December 2014; accepted 22 January 2015; published online 5 February 2015)

\begin{abstract}
We report a comparative synchrotron radiation x-ray diffraction study of $\mathrm{GaAs}_{1-y} \mathrm{~N}_{y}$ microstructures obtained by two different patterning methods: spatially selective $\mathrm{H}$ incorporation achieved by using $\mathrm{H}$-opaque masks and spatially selective $\mathrm{H}$ removal attained by laser writing. These methods are emerging as original routes for fabrication of micro- and nano-structures with in-plane modulation of the bandgap energy. By measuring the out-of-plane and in-plane lattice parameters, we find that for both patterning approaches the largest part of the micro-structure volume remains tensile-strained and pseudomorphic to the substrate, regardless of the compressive-strained hydrogenated barriers. However, a larger lattice disorder is probed in the laser-written micro-structures and attributed to partial removal of $\mathrm{H}$ and/or strain changes at the micro-structure boundaries. This larger lattice disorder is confirmed by photoluminescence studies. (C) 2015 AIP Publishing LLC.
\end{abstract}

[http://dx.doi.org/10.1063/1.4907324]

Innovative devices based on semiconductor nanostructures require full control over the nano-structure size, position, and distribution. ${ }^{1,2}$ The control of material properties (bandgap energy, electronic structure, and strain) in the growth plane is particularly challenging for all the manufacturing approaches presently used. In fact, on the one hand, top-down methods ${ }^{3}$ rely on complex and time-consuming lithography processes, and the optical properties of the resulting nano-structures often suffer from damage inherent to the fabrication method. On the other hand, bottom-up methods based on spontaneous self-assembly ${ }^{4,5}$ can hardly control the spatial arrangement of nano-structures. Recently, some of us have proposed an original route for engineering fundamental electronic properties in the growth plane by exploiting the effects of hydrogen irradiation in $\mathrm{GaAs}_{1-y} \mathrm{~N}_{y} / \mathrm{GaAs}$ epilayers. ${ }^{6}$ In these alloys, the substitution of few percents of As atoms by $\mathrm{N}$ leads to strong, unusual modifications of the host crystal. In particular, it causes a giant reduction in the bandgap energy, thus giving access to wavelengths of interest for many optoelectronics applications. ${ }^{7}$ Post-growth irradiation with atomic hydrogen completely reverses the bandgap red shift caused by $\mathrm{N}$ incorporation: ${ }^{8}$ this is due to the formation of stable $\mathrm{N}-\mathrm{H}$ complexes ${ }^{9}$ which modify, among other parameters, the crystal lattice constant. ${ }^{10,11}$ Therefore, by allowing $\mathrm{H}$ incorporation only in selected regions of the sample, it is possible to achieve a spatially tailored modulation of the bandgap energy as well as of the lattice parameter in the growth plane. This can be done by either deposition of H-opaque masks (hydrogen-masking, $\mathrm{HM}$ ), which impede $\mathrm{H}$ diffusion in defined regions of the crystal, ${ }^{6}$ or photo-dissociation of the N-H complexes in hydrogenated samples by a focused laser beam (laser

a)gianluca.ciatto@synchrotron-soleil.fr writing, LW). ${ }^{12,13}$ Figure 1 shows a sketch of the two alternative patterning methods.

These two fabrication approaches feature different advantages. On the one hand, HM guarantees extremely well defined interfaces between $\mathrm{H}$-treated and $\mathrm{H}$-free regions, with $\mathrm{H}$ (or D) profiles decaying by a factor of ten within $<5 \mathrm{~nm} .{ }^{14}$ On the other hand, LW is faster and more versatile than HM, although less abrupt $\mathrm{H} / \mathrm{no}-\mathrm{H}$ interfaces are expected in this case. In both instances, a reliable, non-destructive investigation of the crystallographic structure and strain state of the fabricated objects

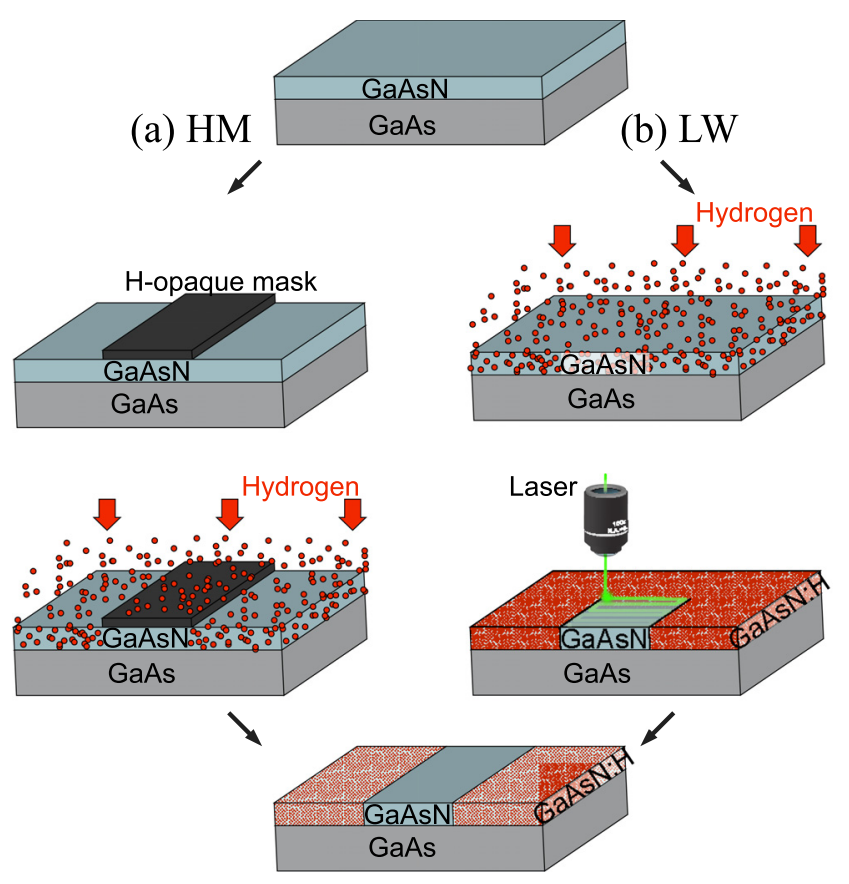

FIG. 1. Sketch of the H-opaque mask (a) and laser writing (b) patterning processes based on hydrogenation of $\mathrm{GaAs}_{1-y} \mathrm{~N}_{y}$. 
is required. Indeed, because of the presence of lateral interfaces between hydrogenated (larger lattice parameter) and nonhydrogenated (smaller lattice parameter) regions, the strain state of the micro- and nano-structures could be modified with respect to that of the original $\mathrm{GaAs}_{1-y} \mathrm{~N}_{y}$ epilayer. To date, strain modifications have been inferred only indirectly by polarization effects on the photoluminescence (PL) signal ${ }^{15}$ and Raman scattering. ${ }^{16}$

In this work, we use Synchrotron Radiation X-ray Diffraction (SXRD) to probe the out-of-plane and in-plane lattice parameters of $\mathrm{GaAs}_{1-y} \mathrm{~N}_{y}$ micro-patterns obtained by using the HM and LW approaches. This technique gives direct access to the crystallographic structure and strain state of the micropatterns and is well suited to monitor the influence of the patterning method employed. The high brilliance of synchrotron radiation ${ }^{17}$ compared to conventional x-ray sources allows us to obtain excellent signal to noise ratio in diffraction curves even relatively far from the Bragg's peak maxima and in grazing incidence condition. ${ }^{18-20}$ This is used to study and compare interference fringes, which depend on the interface quality. Here, we discuss the similarities and differences observed in the SXRD patterns taken on HM and LW micro-structures.

We studied two samples cut from the same original $\operatorname{GaAs}_{1-y} \mathrm{~N}_{y} / \mathrm{GaAs}$ epilayer, with $\mathrm{N}$ concentration $y=0.9 \%$ and thickness of $200 \mathrm{~nm}$. Hydrogenation was performed at $300^{\circ} \mathrm{C}$ by irradiating the samples with an $\mathrm{H}$-dose of 2 $\times 10^{18}$ ions $/ \mathrm{cm}^{2}$ by means of a Kaufman ion source. ${ }^{21}$ The first sample was patterned by using a $\mathrm{H}$-opaque mask prior to hydrogenation, while for the second one we performed LW after hydrogenation, as sketched in Fig. 1. Electron beam lithography-defined masks ${ }^{22}$ were patterned out of a $\mathrm{H}$-opaque, negative hydrogen silsesquioxane resist using a Vistec EBPG 5HR machine working at $100 \mathrm{kV}$. For LW, we used a focused laser beam of wavelength $532 \mathrm{~nm}$, power $\mathrm{P}=20 \mathrm{~mW}$, and exposure time $\mathrm{t}=20 \mathrm{~s}$. $\mu \mathrm{PL}$ maps were obtained by using the latter setup and integrating the local PL intensity in the spectral range of interest. Different pattern shapes (triangle, cross, and H-shape) of $5 \times 5 \mu \mathrm{m}^{2}$ inplane dimension were realized (the out of plane dimension is set by the epilayer's thickness). To increase the XRD signal, studies were performed on an array of micro-structures covering a large area of the epilayers (at least $1.8 \times 1.8 \mathrm{~mm}^{2}$ ). The separation of the different elements in the array is of $200 \mu \mathrm{m}$ along the two in-plane directions. Fig. 2(a) shows a SEM image of a selected area of the sample surface with an array of H-opaque masks (in black) on top (first step in Fig. 1(a)). Fig. 2(b) shows SEM images of the three different pattern features, while Fig. 2(c) shows $\mu \mathrm{PL}$ images of the same shapes realized by LW. $\mu \mathrm{PL}$ images illustrate how the emission properties of the epilayer are modified in correspondence of the patterns. SXRD measurements were performed at the SIRIUS beamline of the SOLEIL synchrotron radiation facility, by using a $\mathrm{Si}-(111)$ monochromator and Pt-coated mirrors to reject harmonics. We selected an incident photon energy of $8.5 \mathrm{keV}$. The incident beam illuminated a portion of the sample (about $0.4 \times 1.7 \mathrm{~mm}^{2}$ ) containing several features, therefore averaging the structural information. XRD was measured in Bragg-Brentano geometry ${ }^{17}$ by means of a 6-circle diffractometer and a X-ray Pixel chips with Adaptive Dynamics (XPAD) ${ }^{23}$ 2D detector. The XRD signal was integrated over a region of interest defined around the maximum of the substrate peak.

As already reported for samples of this kind, ${ }^{24}$ the as grown $\mathrm{GaAs}_{1-y} \mathrm{~N}_{y}$ epilayer used in this work is pseudomorphic to the GaAs substrate (in-plane lattice parameter $\mathrm{a}_{/ /}$ $=\mathrm{a}_{\mathrm{GaAs}}=5.653 \AA$ ). Hence, the out-of-plane lattice parameter $\mathrm{a}_{\perp}$ is related to $\mathrm{a}_{/ /}$and to the relaxed lattice parameter $\mathrm{a}_{0}$ by the formula ${ }^{25}$

$$
\mathrm{a}_{\perp}=\mathrm{a}_{0}+2 \frac{\mathrm{C}_{12}}{\mathrm{C}_{11}}\left(\mathrm{a}_{0}-\mathrm{a}_{/ / /}\right),
$$

where $C_{12}$ and $C_{11}$ are material's elastic constants. Since $a_{0}$ is smaller than $\mathrm{a}_{\mathrm{GaAs}}$, the epilayer is tensile strained on the substrate and $\mathrm{a}_{\perp}$ is contracted (as sketched in Fig. 3(a)). Once the patterns are created using one of the two selectivehydrogenation methods described above, the unit cells inside the $\mathrm{GaAs}_{1-y} \mathrm{~N}_{y}$ patterns are subjected to a biaxial stress. In fact, since the lattice parameter of hydrogenated $\mathrm{GaAs}_{1-y} \mathrm{~N}_{y}$ is (a)

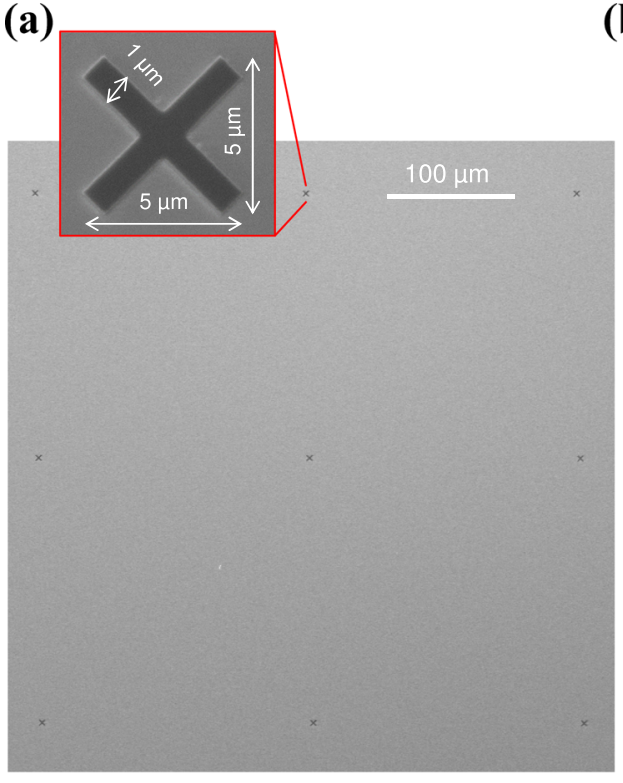

(b)
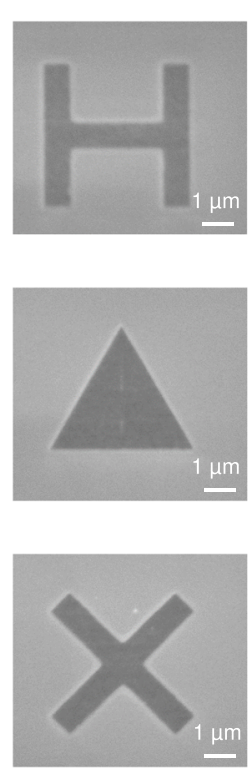

(c)

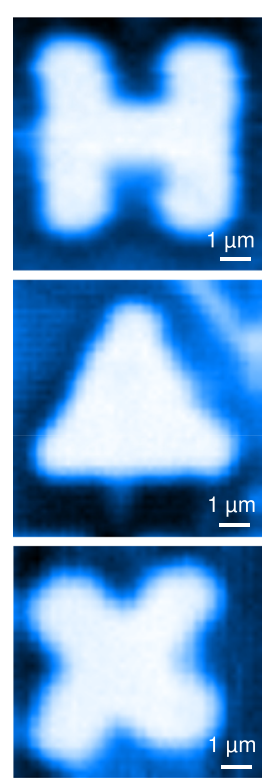

FIG. 2. (a) SEM image of an array of cross-shape H-opaque masks (in black) on top of the sample surface (first step in Fig. 1(a)). (b) SEM images of the three different pattern features. (c) $\mu \mathrm{PL}$ images of the three pattern features realized by LW. The PL maps were obtained by plotting the PL intensity around $1.26 \mathrm{eV}$ (namely, the bandgap energy of $\mathrm{H}$-free $\mathrm{GaAs}_{1-y} \mathrm{~N}_{y}$ ) with laser power $\mathrm{P}=0.5 \mathrm{~mW}$ and wavelength $\lambda=633 \mathrm{~nm}$. 
(a)

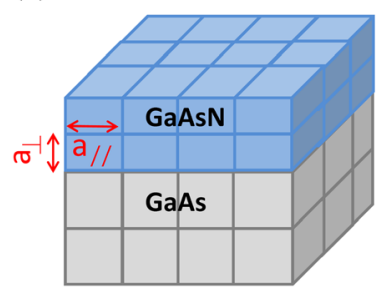

(c)

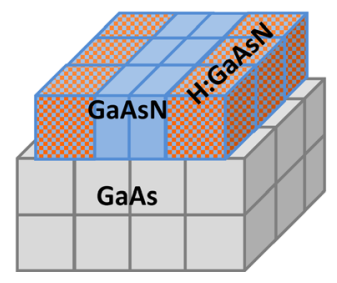

(b)

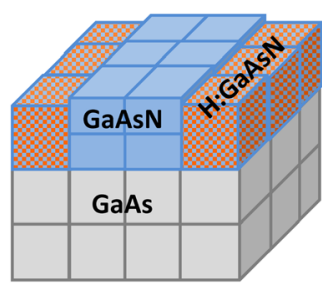

(d)

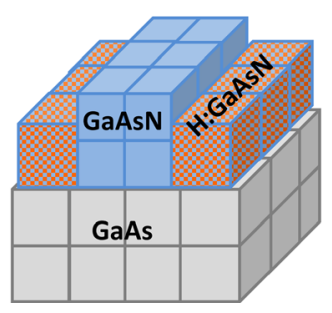

FIG. 3. Sketch of the possible strain conditions for the $\mathrm{GaAs}_{1-y} \mathrm{~N}_{y}$ microstructures, which can be probed by SXRD. The micro-objects are represented by a few unit cells only and the tetragonal distortion is exaggerated for clarity reasons.

larger than that of GaAs, the hydrogenated part of the sample, including the regions surrounding the micro-objects, is subjected to compressive strain. In principle, the unit cells inside the micro-objects can feel and react to the opposite stress generated by the substrate at the bottom and by the lateral hydrogenated barriers. Therefore, the pattern structure can accommodate strain in different ways: (1) remaining pseudomorphic to the substrate (see Fig. 3(b)), (2) adjusting the lattice parameter to match that of the barriers (Fig. 3(c)), or (3) losing coherence with both barriers and substrate (Fig. 3(d)). All intermediate situations between these limit cases cannot be discarded a priori. This motivates a comprehensive XRD study of the patterned epilayers. As described below, SXRD enables us to discriminate between these different scenarios.

In Fig. 4(a), we show $\Theta-2 \Theta$ SXRD curves performed in the vicinity of the [004] crystal plane reflection for the HMand LW-patterned samples along with a reference piece of $\mathrm{GaAs}_{1-y} \mathrm{~N}_{y}$. The reference piece was cut from the pristine epilayer and not hydrogenated. For clarity, the SXRD curve of the reference sample was shifted along the vertical axis. [004] XRD allows direct measurement of $\mathrm{a}_{\perp}$ by application of Bragg's law. In the curve of the reference sample, the difference in angular position between the peaks relative to the substrate (vertical line 1) and $\mathrm{GaAs}_{1-y} \mathrm{~N}_{y}$ epilayer (vertical line 2) is in agreement with the nominal concentration $y=0.9 \%$ and Eq. (1). The presence of well defined and intense Pendellösung fringes ${ }^{26}$ attests to the excellent quality of the interface and the absence of relaxation. In the SXRD curves of the patterned samples, a new peak corresponding to the hydrogenated epilayer (indicated by the vertical line 3) appears on the left of the substrate one. At the same time, the intensity of the $\mathrm{GaAs}_{1-y} \mathrm{~N}_{y}$ peak (vertical line 2) decreases as expected since only the regions inside the patterns are preserved from the lattice parameter expansion caused by hydrogenation. The position of the $\mathrm{GaAs}_{1-y} \mathrm{~N}_{y}$ peak in both HM and LW samples does not change compared to the reference sample. This indicates that the micro-objects (or at least a large fraction of the unit cells they consist of) remain pseudomorphic to the GaAs
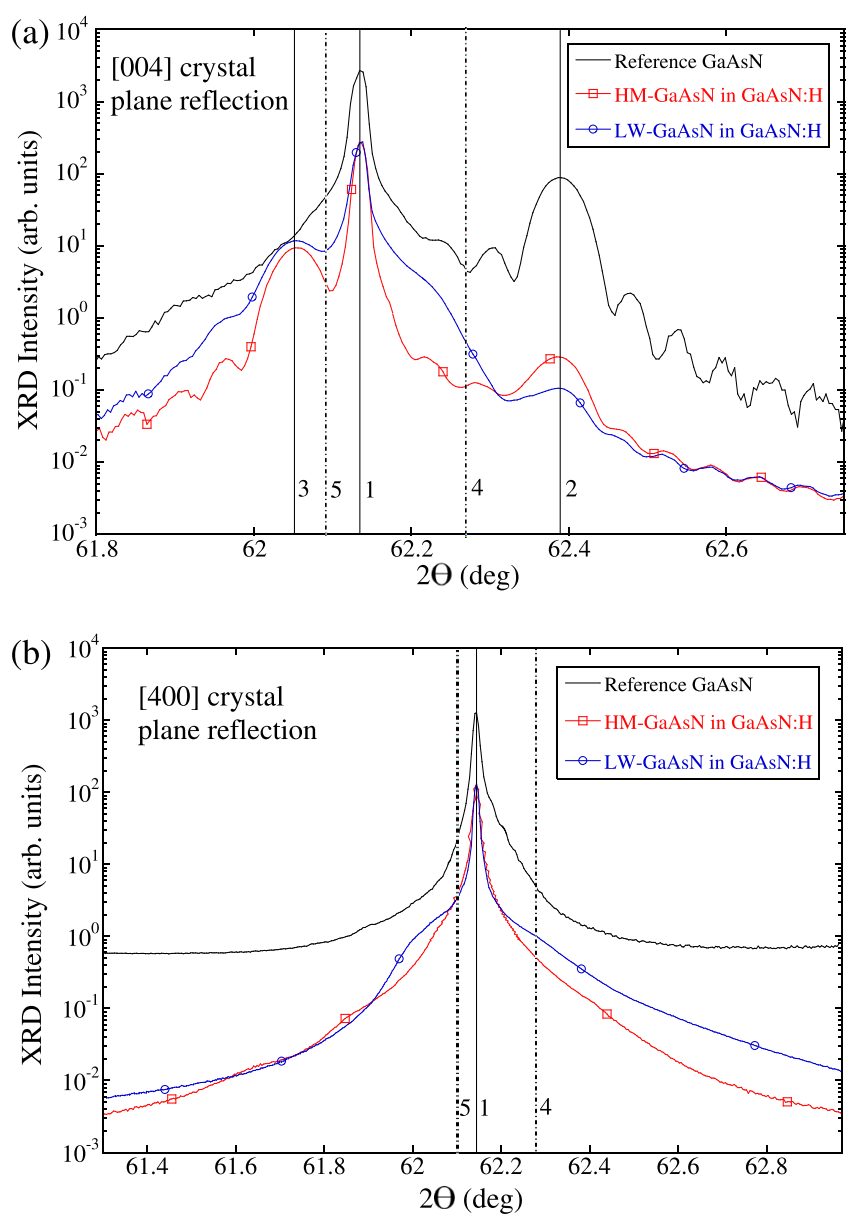

FIG. 4. SXRD $\Theta-2 \Theta$ curves taken around (a) the [004] crystal plane reflection and (b) the [400] reflection for HM- and LW-patterned samples along with a reference $\mathrm{GaAs}_{1-y} \mathrm{~N}_{y}$. For both reflections, the curve of the reference sample has been shifted-up for better visualization.

substrate, regardless of the extended hydrogenated barriers (similar to the sketch in Fig. 3(b)). This is confirmed by the persistence of clear interference fringes, although with reduced intensity. The position of the hydrogenated $\mathrm{GaAs}_{1-y} \mathrm{~N}_{y}$ peak (vertical line 3) is also the same in the HM and LW samples. However, the SXRD curves for the two patterned samples are not identical. The fringe envelope for the HM sample is similar to that of the reference sample all over the curve on the right of the substrate peak. Conversely, for the LW sample, fringes almost disappear in the region between vertical lines 1 and 2, and the curve shape is different. Moreover, the peak corresponding to hydrogenated $\mathrm{GaAs}_{1-y} \mathrm{~N}_{y}$ is broader and less separated from the substrate one. These differences are observed around angles corresponding to the expected peak positions for complete relaxation of the $\mathrm{GaAs}_{1-y} \mathrm{~N}_{y}$ epilayer (vertical line 4, sketch in Fig. 3(d)) and of the hydrogenated $\mathrm{GaAs}_{1-y} \mathrm{~N}_{y}$ (vertical line 5), respectively.

Consistent results are obtained when measuring SXRD in the vicinity of the [400] crystal plane reflection. These measurements, performed at a grazing incidence angle of $0.2^{\circ}$ and shown in Fig. 4(b), give access to a//. Grazing incidence geometry enhances XRD from the epilayer with respect to the substrate. The $\Theta-2 \Theta$ SXRD curves of the two patterned samples show a unique peak corresponding to the one of the reference sample. This confirms that both the hydrogenated 
barriers and the non-hydrogenated patterns in the $\mathrm{GaAs}_{1-y} \mathrm{~N}_{y}$ epilayer (or, at least a large portion of them) are pseudomorphic to the GaAs substrate. However, for the LW-sample, the peak is broader and shoulders on both sides of the maximum are visible. The shoulder on the right of the substrate peak corresponds to the expected position for the $\mathrm{GaAs}_{1-y} \mathrm{~N}_{y}$ peak in case of total lattice relaxation (vertical line 4).

The differences observed in the SXRD curves for the HM- and LW-patterned samples can be explained by the larger lattice disorder induced by the LW-technique. Following the photo-dissociation of the N-H complexes by $\mathrm{LW}, \mathrm{H}$-atoms may remain in the lattice as interstitials, thus distorting the lattice and/or promoting the formation of H-defect complexes. ${ }^{27}$ Moreover, since the spatial resolution of LW is of about $1 \mu \mathrm{m}$ (Refs. 12 and 13) and the micropattern lateral size is $5 \mu \mathrm{m}$, SXRD may be sensitive to possible relaxation or strain change in confined regions of the patterns close to the boundaries. For the HM-sample, the relaxed region would be so small (of the order of $10 \mathrm{~nm}$ ) to produce no visible effect in the averaged SXRD curves.

Additional differences between the LW and HM patterns can be seen by comparing the $\mu \mathrm{PL}$ maps and spectra shown in Fig. 5. Fig. 5(a) shows $\mu$ PL maps of H-shape patterns obtained by LW and HM. Typical PL spectra taken at points located inside (A) and outside (B) the pattern, respectively, are shown in Fig. 5(b) and compared with the spectrum taken on the reference sample. The laser spot size is not sufficiently small to provide evidence for the better resolution of the HM-patterning technique ${ }^{14}$ with respect to LW. However, interesting considerations can be made from the analysis of the relative intensity of the $\mathrm{GaAs}_{1-y} \mathrm{~N}_{y}$ and $\mathrm{GaAs}$ PL emission. The $\mathrm{GaAs}_{1-y} \mathrm{~N}_{y}$ PL band is peaked in both patterns at $\sim 1.26 \mathrm{eV}$ (namely, the bandgap energy of as-grown $\mathrm{GaAs}_{1-y} \mathrm{~N}_{y}$ ). However, while the PL spectrum taken inside
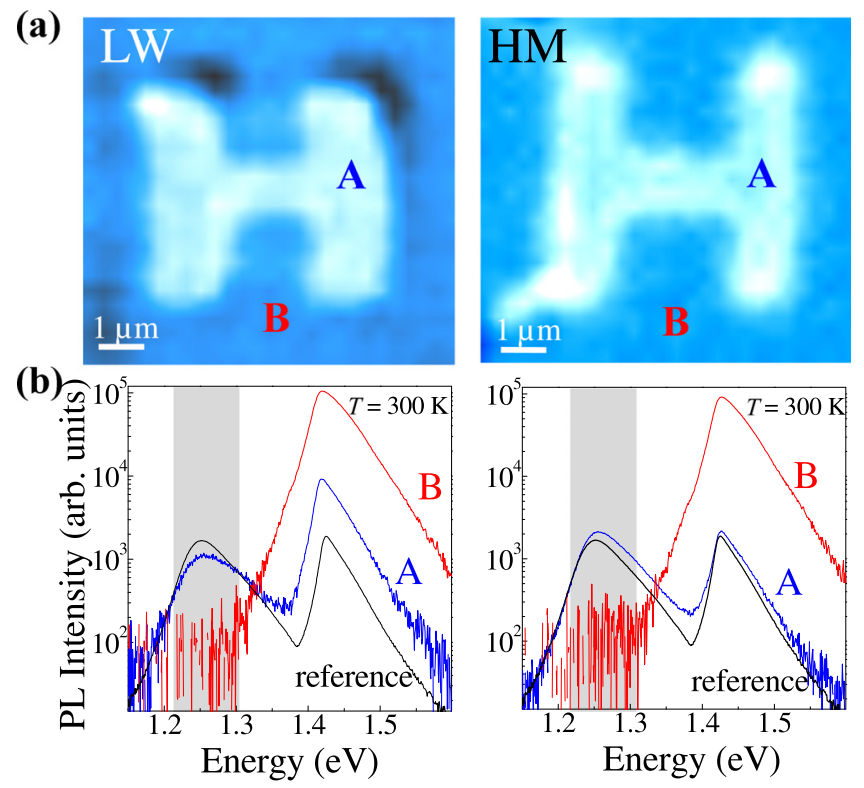

FIG. 5. (a) $\mu \mathrm{PL}$ maps of a LW- and a HM-patterned H-shape and (b) PL spectra taken within (point A) and out of (point B) the H-pattern. A PL spectrum acquired on the reference $\mathrm{GaAs}_{1-y} \mathrm{~N}_{y}$ sample is also shown. The shaded area in (b) indicates the spectral region used to obtain the maps in (a). The PL spectra and maps were acquired with laser power $\mathrm{P}=0.5 \mathrm{~mW}$ and laser wavelength $\lambda=633 \mathrm{~nm}$. the HM pattern is identical to that of the reference sample, in the LW pattern the PL intensity at $\sim 1.26 \mathrm{eV}$ is smaller and that at the GaAs energy $(\sim 1.42 \mathrm{eV})$ is larger. This indicates that in the LW pattern, $\mathrm{H}$ may remain in the GaAs buffer layer beneath and passivate non-radiative recombination centers of GaAs (thus leading to a stronger GaAs PL emission). At the same time, $\mathrm{H}$ can also still be present within the LW region, occupying a different site from the one of the stable N-H complex. ${ }^{9}$ In this new "unwanted" position, it would create non-radiative recombination in $\mathrm{GaAs}_{1-y} \mathrm{~N}_{y}$ thus weakening the $\mathrm{GaAs}_{1-y} \mathrm{~N}_{y}$ PL emission.

In conclusion, we have investigated the average crystal structure and strain of micro-objects obtained by two original patterning techniques based on hydrogenation of $\mathrm{GaAs}_{1-y} \mathrm{~N}_{y}$ epilayers. The micro-structures are basically pseudomorphic to the GaAs substrate, even if an increased structural disorder is observed in patterns obtained by laser writing. This disorder may originate from the formation of defects and/or strain variation in limited regions close to the micro-structure boundaries. The present results provide a reliable feedback for finite element simulations and for the engineering of other interesting micro- and nano-structures (for example, dots into photonic cavities and nano-rings) using the hydrogenation approach. ${ }^{28}$

We thank Synchrotron SOLEIL for general facilities placed at our disposal. The experiment at the SIRIUS beamline was performed during in-house research time. We are grateful for technical support by Mr. N. Aubert. We acknowledge S. Rubini and F. Martelli for providing the samples. G.P., M.F., and A.Po. acknowledge funding from the Italian MIUR (under FIRB project DeLIGHTeD). A.Po. acknowledges funding by Sapienza Università di Roma under the "Ateneo 2013" grant.

${ }^{1}$ T. Yoshie, A. Scherer, J. Hendrickson, G. Khitrova, H. M. Gibbs, G. Rupper, C. Ell, O. B. Shchekin, and D. G. Deppe, Nature 432, 200 (2004).

${ }^{2}$ D. Englund, B. Shields, K. Rivoire, F. Hatami, J. Vuckovic, H. Park, and M. D. Lukin, Nano Lett. 10, 3922 (2010).

${ }^{3}$ C. Gréus, L. Butov, F. Daiminger, A. Forchel, P. A. Knipp, and T. L. Reinecke, Phys. Rev. B 47, 7626(R) (1993).

${ }^{4}$ D. Bimberg, M. Grundmann, and N. N. Ledentsov, Quantum Dot Heterostructures (Wiley, Chichester, 1998).

${ }^{5}$ X. Wang, X. Ren, K. Kahen, M. A. Hahn, M. Rajeswaran, S. Maccagnano-Zacher, J. Silcox, G. E. Cragg, A. L. Efros, and T. D. Krauss, Nature 459, 686 (2009).

${ }^{6}$ S. Birindelli, M. Felici, J. S. Wildmann, A. Polimeni, M. Capizzi, A. Gerardino, S. Rubini, F. Martelli, A. Rastelli, and R. Trotta, Nano Lett. 14, 1275 (2014), and references therein.

${ }^{7}$ M. Henini, Dilute Nitride Semiconductors (Elsevier, Oxford, UK, 2005).

${ }^{8}$ A. Polimeni, G. Baldassarri Höger von Högersthal, M. Bissiri, M. Capizzi, M. Fischer, M. Reinhardt, and A. Forchel, Phys. Rev. B 63, 201304R (2001).

${ }^{9}$ G. Ciatto, F. Boscherini, A. Amore Bonapasta, F. Filippone, A. Polimeni, M. Capizzi, M. Berti, G. Bisognin, D. De Salvador, L. Floreano et al., Phys. Rev. B 79, 165205 (2009).

${ }^{10}$ A. Polimeni, G. Ciatto, L. Ortega, F. Jiang, F. Boscherini, F. Filippone, A. Amore Bonapasta, M. Stavola, and M. Capizzi, Phys. Rev. B 68, 085204 (2003).

${ }^{11}$ M. Berti, G. Bisognin, D. De Salvador, E. Napolitani, S. Vangelista, A. Polimeni, M. Capizzi, F. Boscherini, G. Ciatto, S. Rubini et al., Phys. Rev. B 76, 205323 (2007).

${ }^{12}$ N. Balakrishnan, A. Patanè, O. Makarovsky, A. Polimeni, M. Capizzi, F. Martelli, and S. Rubini, Appl. Phys. Lett. 99, 021105 (2011). 
${ }^{13}$ N. Balakrishnan, G. Pettinari, O. Makarovsky, L. Turyanska, M. W. Fay, M. De Luca, A. Polimeni, M. Capizzi, F. Martelli, S. Rubini et al., Phys. Rev. B 86, 155307 (2012).

${ }^{14}$ R. Trotta, A. Polimeni, M. Capizzi, D. Giubertoni, M. Bersani, G. Bisognin, M. Berti, S. Rubini, F. Martelli, L. Mariucci et al., Appl. Phys. Lett. 92, 221901 (2008).

${ }^{15}$ R. Trotta, A. Polimeni, M. Capizzi, F. Martelli, S. Rubini, M. Francardi, A. Gerardino, and L. Mariucci, Appl. Phys. Lett. 94, 261905 (2009).

${ }^{16}$ M. Geddo, E. Giulotto, M. S. Grandi, M. Patrini, R. Trotta, A. Polimeni, M. Capizzi, and F. Martelli, Appl. Phys. Lett. 101, 191908 (2012).

${ }^{17}$ S. Mobilio, F. Boscherini, and C. Meneghini, Synchrotron Radiation. Basics, Methods and Applications (Springer, 2015).

${ }^{18}$ T. Metzger, V. Favre-Nicolin, G. Renaud, H. Renevier, and T. Schülli, "Nanostructures in the light of synchrotron radiation: Surface-sensitive X-ray techniques and anomalous scattering," in Characterization of Semiconductor Heterostructures and Nanostructures, 1st ed., edited by C. Lamberti (Elsevier, Amsterdam, 2008).

${ }^{19}$ U. Pietsch, V. Holy, and T. Baumbach, High-Resolution X-Ray Scattering. From Thin Films to Lateral Nanostructures (Springer, Berlin, 2004).
${ }^{20}$ O. H. Seeck and B. Murphy, X-Ray Diffraction. Modern Experimental Techniques (Pan Stanford, 2014).

${ }^{21}$ A. Kaufman, Rev. Sci. Instrum. 61, 230 (1990).

${ }^{22}$ A. A. Tseng, K. Chen, C. D. Chen, and K. J. Ma, IEEE Trans. Electron. Packag. Manuf. 26, 141 (2003).

${ }^{23}$ K. Medjoubi, T. Bucaille, S. Hustache, J.-F. Bérar, N. Boudet, J.-C. Clemens, P. Delpierre, and B. Dinkespiler, J. Synchrotron Radiat. 17, 486 (2010).

${ }^{24}$ G. Bisognin, D. De Salvador, C. Mattevi, M. Berti, A. Drigo, G. Ciatto, L. Grenouillet, P. Duvaut, P. Gilet, and H. Mariette, J. Appl. Phys. 95, 48 (2004).

${ }^{25}$ J. Hornstra and W. J. Bartels, J. Cryst. Growth 44, 513 (1978).

${ }^{26}$ N. Kato and A. R. Lang, Acta Crystallogr. 12, 787 (1959).

${ }^{27}$ L. Wen, M. Stavola, W. B. Fowler, R. Trotta, A. Polimeni, M. Capizzi, G. Bisognin, M. Berti, S. Rubini, and F. Martelli, Phys. Rev. B 86, 085206 (2012).

${ }^{28}$ M. Felici, S. Birindelli, R. Trotta, M. Francardi, A. Gerardino, A. Notargiacomo, S. Rubini, F. Martelli, M. Capizzi, and A. Polimeni, Phys. Rev. Appl. 2, 064007 (2014). 